\title{
Prevalência de queda grave e fatores associados em idosos brasileiros: resultados da Pesquisa Nacional de Saúde, 2013
}

\author{
The prevalence of severe falls and associated factors among elderly \\ Brazilians: Results of the 2013 National Health Survey
}

Juleimar Soares Coelho de Amorim (https://orcid.org/0000-0003-3218-1769) ${ }^{1}$ Mary Anne Nascimento Souza (https://orcid.org/0000-0002-3525-6554) ${ }^{1}$ Juliana Vaz de Melo Mambrini (https://orcid.org/0000-0002-0420-3062) ${ }^{2}$ Maria Fernanda Lima-Costa (https://orcid.org/0000-0002-3474-2980) 1,2 Sérgio Viana Peixoto (https://orcid.org/0000-0001-9431-2280) 2,3
${ }^{1}$ Programa de PósGraduação em Saúde Coletiva, Instituto René Rachou, Fiocruz Minas. Av. Augusto de Lima 1715, Barro Preto. 30190-009 Belo Horizonte MG Brasil. sergio.peixoto@fiocruz.br ${ }^{2}$ Núcleo de Estudos em Saúde Pública e Envelhecimento, Instituto René Rachou, Fiocruz Minas. Belo Horizonte MG Brasil.

${ }^{3}$ Departamento de Gestão em Saúde, Escola de Enfermagem, Universidade Federal de Minas Gerais. Belo Horizonte MG Brasil.

\begin{abstract}
The scope of this study was to estimate the prevalence and factors associated with severe falls among elderly Brazilians. It included a transversal study of 10,537 elderly individuals who participated in the National Health Survey (2013). The dependent variable was a report of severe falls in the past year. The independent variables comprised, hierarchically, socio-demographic factors, behavior, health status and functional capacity. Logistic regression was used to obtain the odds ratio (OR) and the confidence interval (95\%CI), using the 13.1 Stata program. The prevalence of severe falls was 7.5\% (95\%CI: 6.7-8.3) and the highest odds were among women (OR: 1.35; 95\%CI: 1.03-1.77), 70 to 79 years of age (OR: 1.47; 95\%CI: $1.12-1.95$ ) and 80 years and above (OR: 2.00; 95\%CI: 1.46-2.74), living without a spouse/partner (OR: 1.37; 95\%CI: 1.08-1.74), sedentary in leisure time (OR: 1.55; 95\%CI: 1.06-2.26), having multimorbities (OR: 1.54; 95\%CI: 1.19-2.00), sleeping difficulties (OR: 2.18; 95\%CI: 1.65-2.88), limitations in daily activities (OR: 2.25; 95\%CI 1.63 -3.10) and use of a walking aid (OR: 1.89; 95\%CI: 1.30-2.73). The prevalence of severe falls was high and multifactorial, enabling the identification of profiles of greater vulnerability, which should be considered in public policies for specific interventions.
\end{abstract}

Key words Accidental falls, Elderly persons, $\mathrm{He}$ alth surveys
Resumo $O$ objetivo foi estimar a prevalência e os fatores associados à queda grave em idosos brasileiros. Estudo transversal, incluindo 10.537 idosos participantes da Pesquisa Nacional de Saúde (2013). A variável dependente foi relato de queda grave último. As variáveis independentes incluiram, hierarquicamente, fatores sociodemográficos, comportamentais, condição de saúde e capacidade funcional. Foi utilizada regressão logística para obter os odds ratio (OR) e os respectivos intervalos de confiança (95\%), utilizando-se o programa Stata 13.1. A prevalência de queda grave foi de 7,5\% (IC95\%: 6,7-8,3) e as maiores chances foram entre mulheres (OR: 1,35; IC95\%: 1,03-1,77), 70 a 79 anos (OR: 1,47; IC95\%: 1,12-1,95) e 80 anos e mais (OR: 2,00; IC95\%: 1,46-2,74), vivendo sem cônjuge (OR: 1,37; IC95\%: 1,08-1,74), sedentários no lazer (OR: 1,55; IC95\%: 1,06-2,26), com multimorbidades (OR: 1,54; IC95\%: 1,192,00), dificuldades no sono (OR: 2,18; IC95\%: 1,65-2,88), limitações em atividades básicas de vida diária (OR: 2,25; IC95\%: 1,63-3,10) e uso de dispositivo para marcha (OR: 1,89; IC95\%: 1,302,73). A prevalência de queda grave foi elevada $e$ multifatorial, permitindo a identificação de perfis de maior vulnerabilidade, o que deve ser considerado no direcionamento de políticas públicas para intervenções específicas.

Palavras-chave Acidentes por Quedas, Idoso, Inquéritos Epidemiológicos 


\section{Introdução}

As quedas graves em idosos tem recebido atenção especial em termos de pesquisas epidemiológicas e na prática clínica, seja por suas repercussões para a saúde individual e coletiva ou pelos desafios na proposição de estratégias de prevenção e reabilitação ${ }^{1-3}$. Em todo o mundo, metade das quedas em idosos resultam em algum tipo de lesão, mas apenas $10,0 \%$ deles referem quedas graves e $1,0 \%$ sofre fratura de quadril ou fêmur ${ }^{4,5}$; já no Brasil a fratura por queda chega a $11,0 \%$ entre idosos ${ }^{6}$. A procura por serviços de saúde relacionada à queda é um sinalizador de gravidade ${ }^{7-9}$, mas existem poucos estudos de base populacional sobre esse evento ${ }^{10-13}$. As consequências das quedas graves incluem a própria hospitalização ${ }^{3}$, mas também medo de nova queda, restrição na participação social e morte ${ }^{5,14}$. Apesar disso, já se sabe que muitos dos seus determinantes são modificáveis com intervenções pertinentes, reduzindo os custos após o evento ${ }^{5,6,10}$.

Os estudos sobre a distribuição e fatores associados às quedas as categorizam como evento acidental ou como resultado do declínio funcional ${ }^{11,12,15-18}$. Embora algumas quedas possam ter causa única, a maioria é marcada por circunstâncias multifatoriais, sejam intrínsecas (idade avançada, fragilidade, presença de múltiplas doenças, déficit visual ou limitação na marcha), extrínsecas (riscos ambientais e comportamentos de risco) ou, na maioria das vezes, pela combinação desses fatores ${ }^{18}$. Por outro lado, as quedas graves predominam em idosos frágeis, em idade avançada, com declínio funcional estabelecido, com múltiplas doenças e usuários de polifarmá$\mathrm{cia}^{7,9,11,12,19-22}$, diferentemente das quedas em geral que ocorrem devido ao comportamento de risco e circunstâncias ambientais que desafiam o equilíbrio corpóreo ${ }^{18}$.

As investigações populacionais discutem as lesões relacionadas à queda grave como importante indicador de gravidade e consequente procura por serviços de saúde ${ }^{8,21}$. No Brasil, os dados nacionais divulgados limitam-se às quedas com fratura $^{6}$, enquanto em outros países são reportados os resultados da queda grave entre idosos, tanto para ocorrência de fratura quanto por procura aos serviços de saúde $7,9,11,21-24$. Assim, no contexto de vida da população idosa brasileira é premente conhecer a prevalência e a distribuição dos fatores de risco de queda grave, evidenciando a necessidade de investigar a busca por assistência à saúde como consequência da queda. Nesse sentido, o objetivo deste estudo foi estimar a prevalência e os fatores associados às quedas graves em idosos brasileiros, a partir dos dados da Pesquisa Nacional de Saúde (2013).

\section{Métodos}

\section{População de estudo}

A Pesquisa Nacional de Saúde (PNS) realizada em 2013 e 2014 foi desenvolvida por uma parceria entre a Secretaria de Vigilância em Saúde do Ministério da Saúde (SVS/MS), a Fundação Oswaldo Cruz (Fiocruz) e o Instituto Brasileiro de Geografia e Estatística (IBGE), cujo objetivo principal foi de produzir dados sobre a situação de saúde e os estilos de vida da população brasileira e dados sobre a atenção à saúde, no que se refere ao acesso e uso dos serviços de saúde, às ações preventivas, à continuidade dos cuidados e ao financiamento da assistência de saúde ${ }^{25}$. A PNS foi aprovada pela Comissão Nacional de Ética em Pesquisa (CONEP) em 2013, e todos os participantes assinaram um termo de consentimento livre e esclarecido ${ }^{26}$.

A PNS é, portanto, um estudo transversal cuja amostra se deu por conglomerados a partir de três estágios de seleção, os setores censitários foram a unidade primária, os domicílios a unidade secundária e os moradores a unidade terciária, representando, portanto, a população adulta brasileira. Foi definido como abrangência geográfica da pesquisa todo o território nacional, incluindo zona urbana e rural e excluindo-se aldeias indígenas, quartéis, bases militares, alojamentos, acampamentos, embarcações, penitenciárias, colônias penais, presídios, cadeias, asilos, orfanatos, conventos e hospitais, pelos quais são áreas com características especiais e com pouca população. Para cada domicílio selecionado, foi entrevistado um adulto com 18 anos ou mais, que foi sorteado de forma equiprobabilística entre todos os adultos residentes no domicílio. Para o presente estudo foram selecionados os participantes com idade maior ou igual a 60 anos, que possuíam informações completas para todas as variáveis de interesse, o que resultou em uma amostra de10.537 idosos analisados (94,3\% dos idosos participantes). A coleta dos dados foi feita por meio de questionários estruturados e medidas antropométricas, ambas foram realizadas no domicílio do participante ${ }^{25}$. 


\section{Variáveis e procedimentos de coleta}

A variável dependente deste estudo foi o relato de queda grave, cuja informação foi obtida por meio da pergunta "Nos últimos 12 meses, teve alguma queda que o(a) levou a procurar o serviço de saúde?", respondida pelo idoso ou informante próximo. Assim, para aqueles que reportaram a ocorrência de queda, verificou-se como consequência do evento a ocorrência de fratura (quadril ou fêmur) e a necessidade de cirurgia.

As variáveis independentes foram selecionadas de acordo com a literatura sobre fatores associados à queda ${ }^{3,7,12,14,18,27-29} \mathrm{e}$ foram agrupadas em três níveis: i) distal - características sociodemográficas (idade, sexo, cor/raça e situação conjugal autorreferidos); ii) intermediário - comportamentos e condição de saúde (tabagismo, consumo de álcool, atividade física, multimorbidade, dificuldades no sono, estado nutricional e problemas na coluna); e, iii) proximal - capacidade funcional (percepção de dificuldade auditiva e visual, limitações nas atividades básicas de vida diária e uso de dispositivo para marcha).

Foram considerados tabagistas atuais aqueles que referiram fumar algum produto do tabaco, no momento da entrevista, e como ex-fumante aqueles que haviam fumado em qualquer momento da vida e haviam parado de fumar esses produtos na ocasião da entrevista. O consumo de risco de álcool foi definido pela ingestão de sete ou mais doses semanais para mulheres e 14 ou mais doses semanais para homens ${ }^{30}$, o consumo leve/moderado foi considerado como qualquer consumo inferior a estes valores e foram classificados como abstémicos aqueles idosos que referiram nunca ter ingerido algum tipo de bebida alcoólica. A prática de atividade física no lazer foi mensurada com base nas respostas às questões sobre frequência semanal e duração da prática de atividade física, nos dias em que era realizada. Foram classificados como suficientemente ativos no lazer os participantes que praticaram pelo menos 150 minutos/semana de atividades físicas leves ou moderadas, ou pelo menos $75 \mathrm{mi}-$ nutos/semana de atividades físicas vigorosas, ou um combinado de atividades físicas moderadas e vigorosas totalizando 150 minutos semanais ${ }^{31}$.

A multimorbidade foi definida pelo relato de diagnóstico médico para duas ou mais doenças/ condições crônicas dentre as seguintes: hipertensão arterial; diabetes; doença do coração (infarto, angina, insuficiência cardíaca); acidente vascular cerebral; câncer e doença no pulmão (enfisema pulmonar, bronquite crônica ou doença pul- monar obstrutiva crônica). Os hábitos do sono nas duas semanas que antecederam a entrevista foram avaliados por meio do relato sobre problemas de sono, que incluíram dificuldade para adormecer, acordar frequentemente à noite ou dormir mais do que o costume. A dificuldade de sono foi atribuída àqueles que referiram pelo menos um dos problemas anteriores em mais da metade dos dias na semana. Foram considerados com problemas na coluna aqueles que informaram ter dor crônica nas costas ou no pescoço, lombalgia, dor ciática e problemas nas vértebras ou disco.

As percepções de dificuldades visual e auditiva foram divididas em três categorias: sem dificuldade (normal), algum distúrbio que não limita as atividades habituais (ruim, mas não limita) e dificuldade visual ou auditiva exacerbada ou que não enxergavam ou escutavam (ruim intensa). A limitação em atividades básicas de vida diária (ABVD) foi atribuída àqueles que informaram ter qualquer dificuldade (alguma, muita ou incapaz) para realizar pelo menos uma entre as seguintes: alimentar-se, tomar banho, usar o banheiro, vestir-se, andar em casa de um cômodo a outro no mesmo andar e deitar-se ou levantarse da cama. Por fim, foi considerado o uso de dispositivos para marcha, tais como bengala, muleta, cadeira de rodas, andador ou qualquer outro equipamento.

No exame físico foram aferidos o peso e a estatura corpórea, de acordo com técnicas padronizadas estando o participante com roupas leves e sem sapatos. Para o peso, utilizou-se uma balança digital e para a altura estadiômetro, ambos portáteis, com os indivíduos em posição vertical ${ }^{26}$. Em seguida, o índice de massa corporal foi calculado como o peso do indivíduo em quilogramas dividido pelo quadrado da altura em metros e os indivíduos foram classificados em baixo peso e eutróficos $\left(<22 \mathrm{~kg} / \mathrm{m}^{2}\right)$ e sobrepeso $\left(\geq 22 \mathrm{~kg} / \mathrm{m}^{2}\right)^{32}$.

\section{Análise estatística}

Todas as variáveis utilizadas nesse estudo foram comparadas entre idosos que referiram e não referiram queda grave, utilizando-se o teste do qui-quadrado de Pearson, visando à seleção das variáveis a serem incluídas no modelo múltiplo. As análises das associações entre variáveis independentes e o desfecho foi baseada em estimativas de odds ratio (OR) e respectivos intervalos de confiança de $95 \%$ pela regressão logística. A análise foi hierarquizada, considerando-se três níveis: distal, intermediário e proximal. As variá- 
veis que apresentaram associações com o evento com valores de $\mathrm{p}<0,20$ na análise univariada foram selecionadas para compor o modelo hierarquizado, sendo mantidas em cada nível aquelas com $\mathrm{p}<0,10$. Assim, as variáveis do nível distal foram ajustadas por aquelas mantidas nesse nível. As do nível intermediário, por variáveis com $\mathrm{p}<0,10$ no nível anterior e por variáveis deste nível, e as do nível proximal, por variáveis com $\mathrm{p}<$ 0,10 no nível anterior e por variáveis deste nível.

Os fatores que apresentaram associações estatisticamente significativas $(\mathrm{p}<0,05)$ com o evento no modelo final foram selecionados como variáveis independentes para ajuste de um modelo de regressão logística para estimar as probabilidades preditas de ocorrência de quedas. Os perfis dos participantes foram definidos pela combinação das respostas para as variáveis independentes. Foram apresentadas as probabilidades preditas, com respectivos intervalos de confiança (95\%), para os três perfis mais prevalentes, além daqueles que apresentaram maior e menor probabilidade de queda. Todas as análises foram realizadas no software Stata ${ }^{\circledR}$ versão 13.0 (StataCorp LLP, CollegeStation, TX), considerando-se a complexidade do plano amostral adotado na PNS e, consequentemente, incorporando os pesos e o efeito de desenho.

\section{Resultados}

A amostra analisada foi constituída por maioria do sexo feminino $(57,4 \%)$, com idade entre $60 \mathrm{e}$ 69 anos (56,3\%), que possuíam cônjuge (42,9\%) e que se auto definiam como brancos $(54,8 \%)$. A maioria dos idosos referiu nunca ter fumado (57,0\%), ser abstêmico ao álcool $(86,0 \%)$, insuficientes em atividade física de lazer $(85,9 \%)$, ter uma ou nenhuma multimorbidade $(62,8 \%)$, não ter dificuldade no sono $(80,5 \%)$, estar com sobrepeso $(86,8 \%)$ e não ter problema na coluna $(71,5 \%)$. Predominaram idosos com percepção de audição $(92,0 \%)$ e visão $(87,1 \%)$ normal, sem limitações em ABVD $(85,0 \%)$ e que não usavam dispositivos auxiliares para marcha (90,7\%). Mais detalhes sobre as características sociodemográficas dos participantes da pesquisa podem ser vistos na Tabela 1 .

A prevalência de queda grave foi de 7,5\% (IC95\%: 6,7-8,3). Entre os que caíram, 10,9\% (IC95\%: 7,2-16,2) referiram fratura de quadril ou fêmur e 12,5\% (IC95\%: 4,3-31,3) necessitaram cirurgia para colocação de prótese. A Figura 1 apresenta a prevalência de queda grave por sexo e faixa etária. Observa-se maiores prevalências de queda grave entre as mulheres $(\mathrm{p}=0,004)$ e entre os mais velhos $(\mathrm{p}<0,001)$.

A Tabela 2 apresenta os resultados da análise univariada da distribuição de queda grave, nos níveis distal, intermediário e proximal. As variáveis sexo, idade, situação conjugal, consumo de álcool, atividade física em lazer, multimorbidades, sono, problemas na coluna percepção da audição, limitação funcional e dispositivo para marcha apresentaram associação estatisticamente significativa $(\mathrm{p}<0,05)$ com a ocorrência de queda grave.

A Tabela 3 apresenta os resultados da análise múltipla da associação entre quedas graves e as variáveis de cada nível da análise hierarquizada. Para a construção dos modelos iniciais, apenas cor de pele, tabagismo e sobrepeso não foram selecionadas para essa etapa, tendo sido mantidas nos modelos as variáveis com valores de $\mathrm{p}$ inferiores a 0,10 , para efeito de ajuste. No nível distal, foram observadas maiores chances de quedas entre as idosas (OR: 1,35; IC95\%: 1,03-1,77), aqueles com 70 a 79 anos (OR: 1,47; IC95\%: 1,12-1,95), 80 anos e mais (OR: 2,00; IC95\%: 1,46-2,74) e que viviam sem cônjuge (OR: 1,37; IC95\%: 1,08-1,74). No nível intermediário, maiores chances de queda grave foram observadas para os idosos insuficientemente ativos no lazer (OR: 1,55; IC95\%: 1,06-2,26), com relato de duas ou mais morbidades (OR: 1,54; IC95\%: 1,19-2,00) e com dificuldades de sono (OR: 2,18; IC95\%: 1,65-2,88). No nível proximal, após ajustamento pelos níveis anteriores e pelas demais variáveis desse nível, maiores chances de queda grave foram observadas entre os idosos com limitação em uma ou mais atividades básicas de vida diária (OR: 2,25; IC95\%: 1,63-3,10) e entre aqueles que reportavam uso de dispositivo para marcha (OR: 1,89; IC95\%: 1,30-2,73).

A Tabela 4 mostra as probabilidades preditas para ocorrência de queda grave, conforme os perfis da população idosa investigada, considerando as variáveis que apresentaram associação significativa com o desfecho na análise anterior. A menor probabilidade de queda foi de 2,3\% (IC95\%: $1,6 \%-3,3 \%$ ), sendo esse perfil composto por homens, de 60 a 69 anos, vivendo com cônjuge, que tinham prática suficiente de atividade física e sem relato de multimorbidade, dificuldade no sono, limitação para ABVD ou uso de dispositivo para marcha. Por outro lado, a maior probabilidade de queda grave foi de 42,1\% (IC95\%: 33,9\%$50,9 \%$ ), estando relacionada às mulheres, com 80 anos e mais, sem cônjuge, com nível insuficiente 
Tabela 1. Características sociodemográficas, comportamentais, condição de saúde e capacidade funcional da amostra de participantes idosos brasileiros de 60 anos ou mais. Pesquisa Nacional de Saúde, 2013.

\begin{tabular}{|c|c|}
\hline Variáveis & $\begin{array}{c}\text { Percentual } \\
(\mathbf{n}=10.537)^{\star}\end{array}$ \\
\hline \multicolumn{2}{|l|}{ Nível Distal } \\
\hline Sexo feminino & 57,4 \\
\hline \multicolumn{2}{|l|}{ Faixa etária (anos) } \\
\hline $60-69$ & 56,3 \\
\hline $70-79$ & 30,0 \\
\hline 80 e mais & 13,7 \\
\hline Sem cônjuge & 42,9 \\
\hline Cor/raça branco & 54,8 \\
\hline \multicolumn{2}{|l|}{ Nível Intermediário } \\
\hline \multicolumn{2}{|l|}{ Tabagismo } \\
\hline Ex-fumante & 31,0 \\
\hline Fumante atual & 11,9 \\
\hline \multicolumn{2}{|l|}{ Consumo de álcool } \\
\hline Leve/moderado & 9,4 \\
\hline Risco & 4,5 \\
\hline Atividade física insuficiente no lazer & 85,9 \\
\hline Duas ou mais multimorbidades & 37,2 \\
\hline Dificuldades de sono & 19,5 \\
\hline Sobrepeso & 86,8 \\
\hline Problemas na coluna & 28,5 \\
\hline \multicolumn{2}{|l|}{ Nível Proximal } \\
\hline \multicolumn{2}{|l|}{ Percepção da audição } \\
\hline Ruim, mas não limita & 7,1 \\
\hline Ruim intensa & 0,1 \\
\hline \multicolumn{2}{|l|}{ Percepção da visão } \\
\hline Ruim, mas não limita & 10,8 \\
\hline Ruim intensa & 2,1 \\
\hline $\begin{array}{l}\text { Limitação funcional em uma ou mais } \\
\text { ABVD }\end{array}$ & 15,0 \\
\hline $\begin{array}{l}\text { Uso de dispositivo auxiliar para } \\
\text { marcha }\end{array}$ & 9,3 \\
\hline \multicolumn{2}{|c|}{$\begin{array}{l}\text { ABVD: atividades básicas de vida diária (alimentar-se, tomar } \\
\text { banho, usar o banheiro, vestir-se, andar em casa de um cômodo } \\
\text { a outro no mesmo andar e deitar-se ou levantar-se da cama); } \\
\text { Porcentagens estimadas considerando o peso individual e } \\
\text { parâmetros amostrais. }{ }^{*} \text { Tamanho amostral, sem considerar } \\
\text { ponderação e efeito de delineamento. }\end{array}$} \\
\hline
\end{tabular}

de atividades físicas no lazer, com multimorbidade, dificuldades no sono, limitação em ABVD e uso de dispositivo para marcha.

\section{Discussão}

Os resultados deste estudo mostraram prevalência de queda grave igual a 7,5\% e os fatores associados foram sexo, faixa etária, situação conjugal, atividade física no lazer, multimorbidades, dificuldades no sono, limitações em ABVD e uso de dispositivo para marcha. Esses fatores permitiram traçar os perfis com menor e maior probabilidade predita para queda grave, podendo auxiliar na determinação dos grupos com maior vulnerabilidade de sofrer esse desfecho e, consequentemente, favorecer o direcionamento de medidas de prevenção do agravo e promoção da saúde.

Quando se considera definições semelhantes ao usado no presente estudo, a prevalência de queda grave, ou seja, aquela que levou à procura por serviços de saúde, apresenta ampla variação entre populações ${ }^{10-13}$. Menores prevalências foram reportadas entre residentes no Canadá (5,8\%), Estados Unidos (11,0\%), Austrália $(11,9 \%)$ e em países europeus $(15,0 \%)$, enquanto maiores prevalências foram observadas entre idosos no Equador (30,6\%) e Taiwan $(34,1 \%)^{7,9,11,21-24}$. A prevalência de fratura de quadril também apresentou variações entre os que tiveram quedas graves, estando entre 1,9\% na Austrália, 2,7\% em Taiwan e 11,0\% no Brasil ${ }^{6,23,24}$. Essas diferenças entre as prevalências podem ser atribuídas aos diferentes contextos em que essas populações vivem, incluindo diferenças de acesso aos serviços de saúde de registro das quedas, o que poderia influenciar nas estatísticas apresentadas, e também às construções, regiões de moradia (proporção da população residente nas zonas rural ou urbana) e até mesmo à disparidade da distribuição de fatores de risco entre as populações, o que também poderia levar a variação no risco de quedas e, consequentemente, a diferenças na prevalência do evento ${ }^{21,33}$.

Em relação aos fatores associados, entre as características sociodemográficas avaliadas, a queda grave foi associada com faixa etária elevada, sexo feminino e não ter cônjuge, o que é consistente com outros estudos de base populacional ${ }^{5,7,34}$. A idade por si só é fator explicativo para ocorrência de quedas em diversos estudos en,6,16,35 $^{\text {e isso pode }}$ ser explicado por alterações biológicas associadas ao envelhecimento, como o estabelecimento da osteoporose, doenças articulares, fraqueza muscular, perda de massa magra, alterações posturais, redução da capacidade de resposta rápida e eficaz do equilíbrio, comprometimento do desempenho das habilidades motoras, marcha e estabilidade postural, que tornam o indivíduo vulnerável à queda ${ }^{18,36}$.

Embora outro estudo tenha demonstrado que o relato de quedas entre homens é menor ${ }^{22}$, 


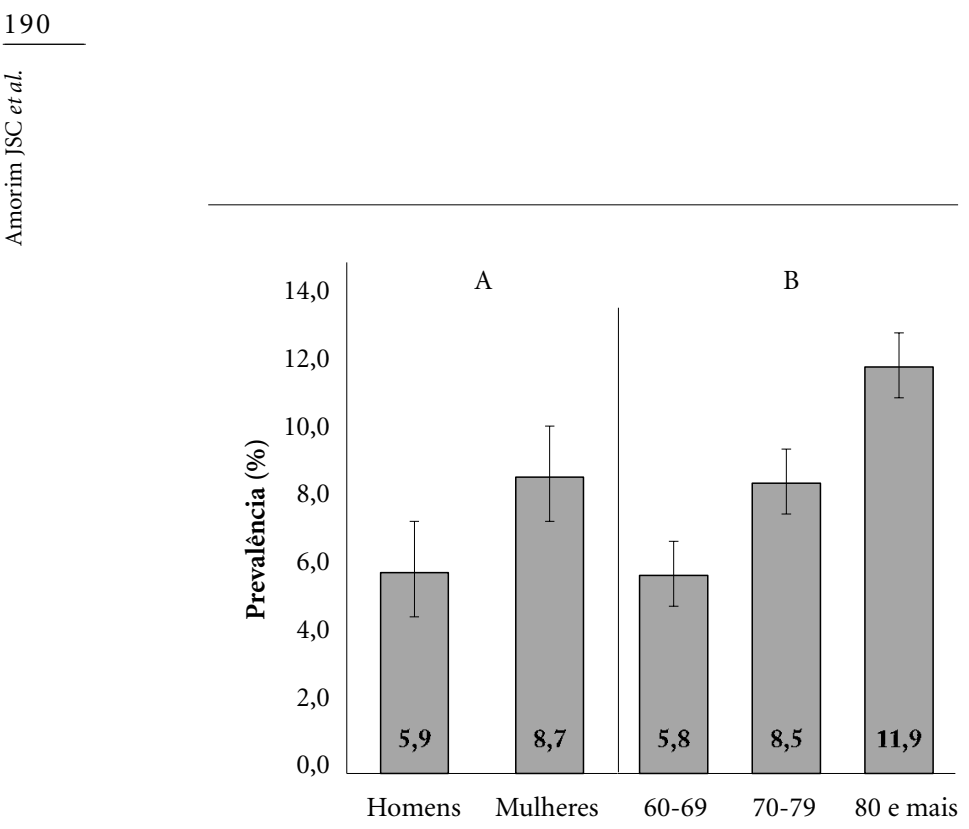

Figura 1. Prevalência de queda grave, de acordo com o sexo (A) e faixa etária (B), da amostra de participantes de idosos brasileiros de 60 anos ou mais. Pesquisa Nacional de Saúde, 2013.
Já foi discutido na literatura que idosos com nível insuficiente de atividade física tendem a ser mais velhos, frágeis, tem maior número de comorbidades, usam mais medicamentos e se movimentam com menos frequência, além de terem reduzido o número de capilares e fibras musculares (massa muscular), limitando a contração muscular, o controle do equilíbrio corpóreo e a capacidade funcional ${ }^{43}$, sugerindo relação intrínseca entre o nível de atividade física e o grau de exposição para quedas ${ }^{4,45}$. Os resultados aqui apresentados, portanto, reforçam a hipótese de associação dessa prática insuficiente e quedas graves, corroborando para uma espiral descendente da função física em idosos com níveis insuficientes de atividade física que resulta em busca aos serviços de saúde devido às quedas.

A multimorbidade referida pelos idosos foi também associada à queda grave nessa população. Esse resultado é consistente com investigações anteriores ao analisar doenças específicas como potencial contribuição para ocorrência de quedas, seja pela via da incapacidade funcional, vulnerabilidade biológica, uso de medicamentos ou até mesmo pelo próprio envelhecimento ${ }^{5,7,21,46}$. Estudo prévio indicou prevalências de quedas até quatro vezes mais elevada em idosos com doenças crônicas e esse resultado encontrase na mesma direção ao verificado neste estudo. Essa relação pode estar associada tanto à presença de sintomas característicos de doenças e suas complicações, quanto a mudanças implementadas em função das mesmas, como maior número de idas aos serviços de saúde e internações, levando a maior chance de queda ${ }^{47}$.

Nesta análise, dificuldades no sono também foi identificada como fator associado à ocorrência de queda grave mesmo após ajuste por variáveis como atividade física, consumo de bebida alcoólica, dor na coluna e multimorbidades. Esse resultado foi semelhante a outro estudo de quedas ${ }^{16}$, no qual a má qualidade do sono foi significativamente associada com maiores chances de ter alguma queda. Assim, os resultados apresentados aqui acrescentam ao conhecimento já produzido a evidência que essa associação também é observada para quedas graves. A qualidade do sono ruim, superficial, fragmentado e de menor eficiência, repercute em sonolência diurna e uso de medicamentos, aumentando, portanto, o risco de eventos graves como as quedas ${ }^{20}$. Parece haver relação entre duração do sono com quedas, em que um possível efeito dose-resposta entre a relação potencial da duração tanto curta quanto longa de sono estão associadas, revelando uma curva 
Tabela 2. Características sociodemográficas, comportamentais, condição de saúde e capacidade funcional entre idosos brasileiros, de acordo com relato de queda grave. Pesquisa Nacional de Saúde, 2013.

\begin{tabular}{|c|c|c|c|c|}
\hline \multirow[b]{2}{*}{ Variáveis } & \multicolumn{2}{|c|}{ Queda Grave (\%) } & \multirow{2}{*}{$\begin{array}{l}\text { OR não } \\
\text { ajustada } \\
\text { (IC95\%) }\end{array}$} & \multirow{2}{*}{$\begin{array}{c}\text { Valor } \\
\mathbf{p}^{\star \star}\end{array}$} \\
\hline & $\begin{array}{c}\text { Não } \\
(\mathbf{n}=9.665)^{*}\end{array}$ & $\underset{(\mathbf{n}=872)^{*}}{\operatorname{Sim}}$ & & \\
\hline \multicolumn{5}{|l|}{ Nível Distal } \\
\hline Sexo feminino (ref.: masculino) & 56,6 & 66,4 & $1,51(1,14-2,01)$ & 0,004 \\
\hline \multicolumn{5}{|l|}{ Faixa etária (anos) } \\
\hline $60-69$ & 57,3 & 43,8 & 1,00 & $<0,001$ \\
\hline $70-79$ & 29,7 & 34,4 & $1,52(1,16-1,99)$ & \\
\hline 80 e mais & 13,0 & 21,8 & $2,19(1,62-2,96)$ & \\
\hline Sem cônjuge (ref.: com cônjuge) & 42,0 & 54,8 & $1,67(1,32-2,13)$ & $<0,001$ \\
\hline Cor/raça não branco (ref.: branco) & 45,4 & 43,2 & $0,91(0,72-1,16)$ & 0,479 \\
\hline \multicolumn{5}{|l|}{ Nível Intermediário } \\
\hline Tabagismo (ref.: não fumante) & & & & 0,564 \\
\hline Ex-fumante & 30,8 & 33,8 & $1,17(0,88-1,54)$ & \\
\hline Fumante atual & 11,9 & 12,4 & $1,12(0,73-1,73)$ & \\
\hline Consumo de álcool (ref.: abstémico) & & & & 0,007 \\
\hline Leve/moderado & 9,7 & 5,0 & $0,47(0,28-0,80)$ & \\
\hline Risco & 4,6 & 2,8 & $0,55(0,27-1,11)$ & \\
\hline $\begin{array}{l}\text { Atividade física insuficiente no lazer (ref.: suficiente } \\
\text { ativos) }\end{array}$ & 85,4 & 91,8 & $1,92(1,32-2,79)$ & 0,001 \\
\hline Duas ou mais multimorbidades (ref.: nenhuma ou uma) & 36,0 & 51,8 & $1,90(1,49-2,42)$ & $<0,001$ \\
\hline Dificuldades de sono (sim) (ref.: não) & 18,1 & 35,9 & $2,53(1,95-3,29)$ & $<0,001$ \\
\hline Sobrepeso (ref.: eutrófico) & 86,9 & 84,7 & $0,83(0,56-1,24)$ & 0,401 \\
\hline Problemas na coluna (sim) (ref.: não) & 27,8 & 37,3 & $1,54(1,20-1,97)$ & 0,001 \\
\hline \multicolumn{5}{|l|}{ Nível Proximal } \\
\hline Percepção da audição (ref.: normal) & & & & 0,006 \\
\hline Ruim, mas não limita & 6,9 & 9,8 & $1,49(0,97-2,29)$ & \\
\hline Ruim intensa & 0,9 & 2,2 & $2,68(1,44-4,99)$ & \\
\hline Percepção da visão (ref.: normal) & & & & 0,071 \\
\hline Ruim, mas não limita & 10,5 & 14,2 & $1,44(0,99-2,11)$ & \\
\hline Ruim intensa & 2,1 & 3,1 & $1,68(0,89-3,19)$ & \\
\hline $\begin{array}{l}\text { Limitação funcional em uma ou mais ABVD (ref.: sem } \\
\text { limitações) }\end{array}$ & 13,2 & 37,2 & $3,89(2,97-5,09)$ & $<0,001$ \\
\hline $\begin{array}{l}\text { Uso de dispositivo auxiliar para marcha (sim) (ref.: não } \\
\text { usa) }\end{array}$ & 8,0 & 25,4 & $3,93(2,90-5,34)$ & $<0,001$ \\
\hline
\end{tabular}

em "U" e o menor risco de quedas tem sido observado entre sete e oito horas de sono por $\mathrm{dia}^{20}$.

Os resultados observados neste estudo revelam que a limitação em ABVD foi uma das variáveis com associação de maior magnitude do modelo, mesmo após ajustes por variáveis do nível distal e intermediário, tais como o sexo, idade, multimorbidades, atividade física, sono E problemas na coluna. No entanto, a associação entre queda grave e limitações funcionais nas ABVDs tem dupla direcionalidade, pois as quedas representam um forte preditor de incapacidade funcional, pela qual é considerada como evento sentinela para perda de independência e marcador de fragilidade ${ }^{11,34}$. Assim, fica evidente que a ocorrência da queda grave vai além de um evento ocasional, acidental, mas compreende também, desfecho de declínios no funcionamento do corpo, como são vistos em estudos etiológicos para detecção do risco de quedas que incluem os testes clínico-funcionais ${ }^{15}$. De todo modo, as quedas e as limitações funcionais fazem parte de um 
Tabela 3. Fatores associados à queda grave entre idosos brasileiros, segundo os níveis hierárquicos. Pesquisa Nacional de Saúde, 2013.

\begin{tabular}{|c|c|c|}
\hline Variáveis & $\begin{array}{c}\text { OR ajustada } \\
\text { (IC95\%) }\end{array}$ & Valor $\mathrm{p}^{*}$ \\
\hline \multicolumn{3}{|l|}{ Nível Distal } \\
\hline Sexo feminino (ref.: masculino) & $1,35(1,03-1,77)$ & 0,032 \\
\hline Idade em anos (ref.: 60-69) & 1,00 & - \\
\hline $70-79$ & $1,47(1,12-1,95)$ & 0,006 \\
\hline 80 e mais & $2,00(1,46-2,74)$ & $<0,001$ \\
\hline Sem cônjuge (ref.: com cônjuge) & $1,37(1,08-1,74)$ & 0,010 \\
\hline \multicolumn{3}{|l|}{ Nível Intermediário } \\
\hline Consumo de álcool (ref.: abstémico) & 1,00 & - \\
\hline Leve/moderado & $0,61(0,36-1,06)$ & 0,072 \\
\hline Risco & $0,77(0,37-1,62)$ & 0,496 \\
\hline Atividade física insuficiente no lazer (ref.: suficientemente ativos) & $1,55(1,06-2,26)$ & 0,022 \\
\hline Duas ou mais morbidades ${ }^{\star \star}$ (ref.: nenhuma ou uma) & $1,54(1,19-2,00)$ & 0,001 \\
\hline Dificuldade de sono (ref.: não) & $2,18(1,65-2,88)$ & $<0,001$ \\
\hline Problemas na coluna (ref.: não) & $1,25(0,96-1,64)$ & 0,101 \\
\hline \multicolumn{3}{|l|}{ Nível Proximal } \\
\hline Percepção da audição (ref.: normal) & 1,00 & - \\
\hline Ruim, mas não limita & $1,18(0,75-1,86)$ & 0,464 \\
\hline Ruim intensa & $1,40(0,70-2,78)$ & 0,337 \\
\hline Percepção da visão (ref.: normal) & 1,00 & - \\
\hline Ruim, mas não limita & $1,20(0,83-1,74)$ & 0,334 \\
\hline Ruim intensa & $0,63(0,29-1,40)$ & 0,258 \\
\hline Limitação em uma ou mais ABVD (ref.: sem limitações) & $2,25(1,63-3,10)$ & $<0,001$ \\
\hline Uso de dispositivo auxiliar para marcha ${ }^{* \star \star}$ (ref: não usa) & $1,89(1,30-2,73)$ & 0,001 \\
\hline \multicolumn{3}{|c|}{$\begin{array}{l}\text { ABVD: atividades básicas de vida diária (alimentar-se, tomar banho, usar o banheiro, vestir-se, andar em casa de um cômodo a outro } \\
\text { no mesmo andar e deitar-se ou levantar-se da cama). Odds ratio (OR) e intervalo de } 95 \% \text { de confiança (IC95\%) ajustados por todas } \\
\text { as variáveis listadas em cada bloco e pelas variáveis dos blocos superiores. }{ }^{*} \text { Valor p do teste de Wald. }{ }^{*} \text { Multimorbidades: hipertensão } \\
\text { arterial, diabetes, infarto, angina, insuficiência cardíaca, acidente vascular cerebral, câncer, enfisema pulmonar, bronquite crônica, } \\
\text { doença pulmonar obstrutiva crônica. }{ }^{* * *} \text { Dispositivos de marcha: bengala, muleta, cadeira de rodas, andador ou qualquer outro } \\
\text { equipamento. }\end{array}$} \\
\hline
\end{tabular}

Tabela 4. Probabilidades preditas para ocorrência de queda grave em idosos brasileiros, conforme os perfis definidos pelas variáveis associadas. Pesquisa Nacional de Saúde, 2013.

\begin{tabular}{|c|c|c|c|c|c|c|c|c|}
\hline Sexo & $\begin{array}{c}\text { Faixa } \\
\text { etária } \\
\text { (em anos) }\end{array}$ & $\begin{array}{l}\text { Presença } \\
\text { de } \\
\text { cônjuge }\end{array}$ & $\begin{array}{c}\text { Prática suficiente } \\
\text { de atividade } \\
\text { física no lazer }\end{array}$ & $\begin{array}{c}\text { Duas } \\
\text { ou mais } \\
\text { morbidades }^{*}\end{array}$ & $\begin{array}{l}\text { Dificuldade } \\
\text { de sono }\end{array}$ & $\begin{array}{c}\text { Limitação } \\
\text { em } \\
\text { ABVD }^{\star *} \\
\end{array}$ & $\begin{array}{c}\text { Uso de } \\
\text { dispositivo } \\
\text { para marcha }^{* * *}\end{array}$ & $\begin{array}{c}\text { Probabilidade } \\
\text { de queda } \\
\text { (IC95\%) }\end{array}$ \\
\hline \multicolumn{9}{|c|}{$\begin{array}{l}\text { Perfis mais frequentes } \\
\end{array}$} \\
\hline Homem & $60-69$ & Sim & Não & Não & Não & Não & Não & $3,1(2,3-4,2)$ \\
\hline Mulher & $60-69$ & Sim & Não & Não & Não & Não & Não & $3,8(2,9-5,0)$ \\
\hline Mulher & $60-69$ & Não & Não & Não & Não & Não & Não & $4,9(3,8-6,4)$ \\
\hline \multicolumn{9}{|c|}{ Perfis com menor probabilidade de queda } \\
\hline Homem & $60-69$ & Sim & Sim & Não & Não & Não & Não & $2,3(1,6-3,3)$ \\
\hline Homem & $70-79$ & Sim & Sim & Não & Não & Não & Não & $2,8(1,8-4,3)$ \\
\hline Homem & 80 e mais & Sim & Sim & Não & Não & Não & Não & $2,9(1,8-4,6)$ \\
\hline \multicolumn{9}{|c|}{ Perfis com maior probabilidade de queda } \\
\hline Mulher & $60-69$ & Não & Não & Sim & Sim & Sim & Sim & $37,0(28,5-46,5)$ \\
\hline Mulher & $70-79$ & Não & Não & Sim & Sim & Sim & Sim & $41,3(32,4-50,9)$ \\
\hline Mulher & 80 e mais & Não & Não & Sim & Sim & Sim & Sim & $42,1(33,9-50,9)$ \\
\hline
\end{tabular}


amplo campo de pesquisas e preocupações de enorme relevância para a saúde pública, devido as suas prevalências, carga de morbidade, elevado custo social e econômico ${ }^{2,3}$.

Entre as variáveis de capacidade funcional, em nossos resultados, um achado importante foi a associação entre o uso de dispositivos auxiliares de marcha e quedas. Entre aqueles idosos que relataram queda grave, $25,3 \%$ faziam uso de dispositivos de marcha, com incremento de $89,0 \%$ a mais nas chances de queda grave. Embora as bengalas, muletas e andadores façam parte de programas multidimensionais de prevenção de quedas por melhorar a independência funcional, a mobilidade, o equilíbrio, a base de suporte corpóreo e reduzir os efeitos de uma ampla gama de deficiências, o uso desses dispositivos apresenta uma associação com o risco aumentado de quedas tanto no que tange ao seu uso incorreto, quanto ao fato de servir como um marcador de declínio funcional na marcha e estabilidade postural, mas também como consequência de quedas anteriores ${ }^{48,49}$. Estudo populacional prévio identificou que os idosos que procuraram os serviços de saúde faziam uso mais regularmente desses dispositivos, aumentando consideravelmente o número de atendimentos em unidades de prontos-socorros, sendo $87,3 \%$ das quedas relacionadas com andador, $12,3 \%$ com bengala e $0,4 \%$ com ambos os dispositivos ${ }^{49}$. Nesse caso, a associação entre quedas graves e o uso de dispositivo auxiliar para marcha sugere aos profissionais a oportunidade de prescrever o dispositivo, revisar e treinar corretamente.

Além dos fatores associados, os resultados descritos no presente estudo permitiram caracterizar os perfis com maior e menor probabilidade de quedas graves, auxiliando no entendimento da população com diferentes níveis de vulnerabilidade a esse desfecho. Observou-se que os perfis mais frequentes são parecidos com aqueles com menor probabilidade de queda, com predominância dos homes, idosos mais jovens (60 a 69 anos), que residiam com cônjuge e que não reportavam comorbidades, dificuldade no sono, limitação em ABVD e uso de dispositivo para marcha. Por outro lado, foi possível observar, por exemplo, que as mulheres, sem cônjuge, que não praticavam atividade física e que relataram piores condições de saúde representaram o grupo com maior probabilidade de quedas graves, mesmo em idades mais jovens. Como observado em nossos achados e também descrito anteriormente, o aumento nos percentuais de quedas grave ocorre em função do número de fatores de risco ${ }^{21}$.
A maior probabilidade de queda grave revelou ampla variedade de fatores associados e permitiu identificar perfis diferenciados, reforçando a hipótese de multideterminação desse evento. A importância de se compreender esses perfis está relacionada ao fato de que nenhum fator isoladamente é capaz de rastrear a vulnerabilidade para queda grave, considerando a multiplicidade das etiologias, fenótipos e fisiopatologia ${ }^{15,16,18,34}$, mas também que as chances de queda grave podem ser reduzidas ao modificar alguns dos fatores. Essas evidências podem contribuir para o entendimento do mecanismo implicado na ocorrência desse agravo, favorecendo o planejamento de ações voltadas ao acompanhamento individual e coletivo, pelo menos no que se refere ao perfil de fatores modificáveis desse segmento populacional.

Como limitação deste estudo, a natureza transversal não permite estabelecer relações temporais entre a queda grave e as variáveis independentes pesquisadas, podendo algumas dessas associações serem resultado de causalidade reversa. Ainda assim, o delineamento foi adequado à investigação dos objetivos estabelecidos, permitindo estimar a prevalência de queda grave e os perfis com maior e menor probabilidade de ocorrência desse evento. Outra limitação é a possibilidade de que as estimativas de prevalência de queda grave estejam subestimadas devido ao viés de memória entre os idosos estudados. Contudo, esse viés foi minimizado nessa análise devido a procura por serviço de saúde ser um sinalizador de gravidade e contribuir para a representação afetiva da queda. Por fim, é possível que o Inquérito tenha subestimado a frequência da gravidade da queda pelo fato dos idosos terem sido admitidos em instituições de longa permanência ou terem morrido em decorrência de tal gravidade, não sendo assim incluídos nas análises. Ainda assim, a pesquisa teve processo de amostragem bem conduzido e utilizou-se de procedimentos padronizados para a coleta das informações.

O rápido envelhecimento de brasileiros tem reflexos em aumentos na carga e na gravidade de doenças, mas também na ocorrência de eventos adversos em saúde e, consequentemente, procura por serviços assistenciais, tal como a elevada prevalência de quedas graves evidenciada neste estudo. Foi possível identificar múltiplos fatores associados a ocorrência dessas quedas em idosos, sendo a maioria deles modificáveis. A redução na prevalência de queda grave exige esforços na articulação de políticas sociais em todos os níveis de gestão, pois aumento esperado no número de 
idosos com relato de quedas podem sobrecarregar os serviços públicos de saúde e, em virtude desse cenário, reforça-se a importância das intervenções preventivas. As medidas para minimizar o risco de quedas podem ser implementadas por uma equipe multidisciplinar para gerenciamento adequado de doenças e por pesquisadores para

\section{Colaboradores}

JSC Amorim trabalhou na concepção, realização da pesquisa e redação final. MAN Souza trabalhou nas análises estatísticas e redação final. JVM Mambrini trabalhou nas análises estatísticas e redação final. MF Lima-Costa trabalhou na revisão crítica e redação final. SV Peixoto trabalhou na orientação, revisão crítica e redação final. planejar ações públicas coletivas. Os resultados descritos nesse estudo podem subsidiar a formulação das políticas públicas nas áreas de promoção, vigilância e atenção à saúde no Sistema Único de Saúde, alinhadas às estratégias da Política Nacional do Idoso, no Brasil, estabelecendo metas para a redução da carga desse evento.

\section{Referências}

1. Lozano R, Naghavi M, Foreman K, Lim S, Shibuya K, Aboyans V, Abraham J, Adair T, Aggarwal R, Ahn SY, Alvarado M, Anderson HR, Anderson LM, Andrews KG, Atkinson C, Baddour LM, Barker-Collo S, Bartels DH, Bell ML, Benjamin EJ, Bennett D, Bhalla K, Bikbov B, Abdulhak AB, Birbeck G, Blyth F, Bolliger I, Boufous S, Bucello C, Burch M, Burney P, Carapetis J, Chen H, Chou D, Chugh SS, Coffeng LE, Colan SD, Colquhoun S, Colson KE, Condon J, Connor MD, Cooper LT, Corriere M, Cortinovis M, Vaccaro KC, Couser W, Cowie BC, Criqui MH, Cross M, Dabhadkar KC, Dahodwala N, De Leo D, Degenhardt L, Delossantos A, Denenberg J, Des Jarlais DC, Dharmaratne SD, Dorsey ER, Driscoll T, Duber H, Ebel B, Erwin PJ, Espindola P, Ezzati M, Feigin V, Flaxman AD, Forouzanfar MH, Fowkes FGR, Franklin R, Fransen M, Freeman MK, Gabriel SE, Gakidou E, Gaspari F, Gillum RF, Gonzalez-Medina D, Halasa YA, Haring D, Harrison JE, Havmoeller R, Hay RJ, Hoen B, Hotez PJ, Hoy D, Jacobsen KH, James SL, Jasrasaria R, Jayaraman S, Johns N, Karthikeyan G, Kassebaum N, Keren A, Khoo J-P, Knowlton LM, Kobusingye O, Koranteng A, Krishnamurthi R, Lipnick M, Lipshultz SE, Ohno SL, Mabweijano J, MacIntyre MF, Mallinger L, March L, Marks GB, Marks R, Matsumori A, Matzopoulos R, Mayosi BM, McAnulty JH, McDermott MM, McGrath J, Mensah GA, Merriman TR, Michaud C, Miller M, Miller TR, Mock C, Mocumbi AO, Mokdad AA, Moran A, Mulholland K, Nair MN, Naldi L, Narayan KMV, Nasseri K, Norman P, O’Donnell M, Omer SB, Ortblad K, Osborne R, Ozgediz D, Pahari B, Pandian JD, Rivero AP, Padilla RP, Perez-Ruiz F, Perico N, Phillips D, Pierce K, Pope 3rd CA, Porrini E, Pourmalek F, Raju M, Ranganathan D, Rehm JT, Rein DB, Remuzzi G, Rivara FP, Roberts T, De León FR, Rosenfeld LC, Rushton L, Sacco RL, Salomon JA, Sampson U, Sanman E, Schwebel DC, Segui-Gomez M, Shepard DS, Singh D, Singleton J, Sliwa K, Smith E, Steer A, Taylor JA, Thomas B, Tleyjeh IM, Towbin JA, Truelsen T, Undurraga EA, Venketasubramanian N, Vijayakumar L, Vos T, Wagner GR, Wang M, Wang W, Watt K, Weinstock MA, Weintraub R, Wilkinson JD, Woolf AD, Wulf S, Yeh P-H, Yip P, Zabetian A, Zheng Z-J, Lopez AD, Murray CJL, AlMazroa MA, Memish ZA. Global and regional mortality from 235 causes of death for 20 age groups in 1990 and 2010: a systematic analysis for the Global Burden of Disease Study 2010. Lancet 2012; 380(9859):2095-2128. 
2. Stevens JA, Corso OS, Filkelstein EA, Miller TR. The costs of fatal and non-fatal falls among older adults. Inj Prev 2006; 12(5):290-295.

3. Sartini M, Cristina ML, Spagnolo AM, Cremonesi P, Costaguta C, Monacelli F, Garau J, Odetti P. The epidemiology of domestic injurious falls in a community dwelling elderly population: an outgrowing economic burden. Eur J Public Health 2010; 20(5):604-606.

4. Stel VS, Pluijm SMF, Deeg DJH, Smit JH, Bouter LM, Lips P. A classification tree for predicting recurrent falling in community-dwelling older persons. JAGS 2003; 51:1356-1364.

5. Bortolon PC, Andrade CLT, Andrade CAF. O perfil das internações do SUS para fratura osteoporótica de fêmur em idosos no Brasil: uma descrição do triênio 2006-2008. Cad Saúde Pública 2011; 27(4):733-742.

6. Siqueira FV, Fachini LA, Silveira DS, Piccini RX, Tomas E, Thumé E, Silva SM, Dilélio A. Prevalence of falls in elderly in Brazil: a contrywide analysis. Cad Saúde Pública 2011; 27(9):1819-1826.

7. Bchele G, Becker C, Cameron ID, Konig HH, Robinovitch S, Rapp K. Preditors of serious consequences of falls in residential aged care: analysis of more than 70,000 falls from residents of Bavarian nursing homes. J Am Med Dir Assoc 2014; 15(8)559-563.

8. Jiang J, Long J, Ling W, Huang G, Guo X, Su L. Incidence of fall-related injury among old people in mainland China. Arch Gerontol Geriatr 2015; 61:131139.

9. Bohl AA, Fishman PA, Ciol MA, Williams B, LoGerfo J, Phelan EA. A longitudinal analysis of total 3-year healthcare costs for older adults who experience a fall requiring medical care. JAGS 2010; 58:853-860.

10. Soares DS, Mello LM, Silva AS, Martinez EZ, Nunes AA. Fraturas de fêmur em idosos no Brasil: análise espaço-temporal de 2008-20012. Cad Saúde Pública 2014; 30(12):2669-2678.

11. De Vries OJ, Peeters GMEE, Lips P, Deeg DJH. Does frailty predict increased risk of falls and fractures? A prospective population-based study. Osteoporos Int 2013; 24:2397-2403

12. Ku YC, Liu ME, Tsai YF, Liu WC, Lin SL, Tsai SJ. Associated factors for falls, recurrent falls, and injurious falls in aged men living in Taiwan veterans homes. Int J Gerontol 2013; 7:80-84.

13. Morrison A, Fan T, Sen SS, Weisenfluh. Epidemiology of falls and osteoporotic fractures: a systematic review. Clinicecon Outcomes Res 2013; 5:9-18.

14. Muraki S, Akune T, Ishimoto Y, Nagata K, Yoshida M, Tanaka S, Oka H, Kawaguchi H, Nakamura K, Yoshimura N. Risk factors for falls in a longitudinal population-based cohort study of Japanese men and women: the ROAD Study. Bone 2013; 52(1):516-523.

15. Vieira LS, Gomes AP, Bierhals IO, Farías-Antúnez S, Ribeiro CG, Miranda VIA, Lutz BH, Barbosa-Silva TG, Lima NP, Bertoldi AD, Tomasi E. Quedas em idosos no Sul do Brasil: prevalência e determinantes. Rev Saúde Pública 2018; 52:22.

16. Prato SCF, Andrade SM, Cabrera MAS, Dip RM, Santos HG, Dellaroza MSG, Mesas AE. Frequência e fatores associados a quedas em adultos com 55 anos e mais. Rev Saúde Pública 2017; 51:37.
17. Okubo Y, Seino S, Yabushita N, Osuka Y, Jung S, Nemoto M, Figueroa R, Tanaka K. Longitudinal association between habitual walking and fall occurrences among community-dwelling older adults: analyzing the different risks of falling. Arch Gerontol Geriatr 2015; 60(1):45-51.

18. Deandrea S, Lucenteforte E, Bravi F, Foschi R, Vecchia CL, Negri E. Risc factors for falls community-dweling older people: a systematic review and meta-analysis. Epidemiology 2010; 21(5):658-668.

19. Lam C, Kang JH, Lin HY, Huang HC, Wu CC, Chen PL. First fall-related injuries requiring hospitalization increase the risk of recurrent injurious falls: a nationwide cohort study in Taiwan. Plos One 2016; 22(11):e0149887.

20. Wu TY, Chie WC, Yang RS, Kuo KL, Wong WK, Liaw CK. Risk factores for single and recurrent falls: a prospective study of falls in community dwelling seniors without cognitive impairment. Prev Med 2013; 57:511-517.

21. Orces $\mathrm{CH}$. Prevalence and determinants of falls among older adults in Ecuador: an analysis of the SABE I Survey. Curr Gerontol Geriatr Res 2013; 495468.

22. Chang VC, Do MT. Risk factors for falls among seniors: implications of gender. Am J Epidemiol 2015; 181(17):21-31.

23. Tsai LY, Tsay SL, Hsieh RK, Yu S, Tsai JM, Chien HH et al. Fall injuries and related factors of elderly patients at a medical Center in Taiwan. Inter J Gerontol 2014; 8(4):203-208.

24. Nilsson M, Eriksson J, Larsson B, Odén A, Johansson $\mathrm{H}$, Lorentzon M. Fall risk assessment predicts fall-related injury, hip fracture, and head injury in older adults. J Am Geriatric Soc 2016; 64(11):2242-2250.

25. Souza-Junior PRB, Freitas MPS, Antonaci GAS, Célia L. Sampling design for the National Health Survey, 2013. Epidemiol Serv Saúde 2015; 24(2):207-216.

26. Instituto Brasileiro de Geografia e Estatística (IBGE). Pesquisa Nacional de Saúde: Manual de antropometria [Internet]. 2013 [acessado Jun 26]. Disponível em: https://www.pns.icict.fiocruz.br/arquivos/Novos/Manual\%20de\%20Antropometria\%20PDF.pdf

27. Coutinho ESF, Bloch KV, Rodrigues LC. Characteristics and circumstances of falls leading to severe fractures in elderly people in Rio de Janeiro, Brazil. Cad Saúde Pública 2009; 25(2):455-459.

28. Wu L, Sun D. Sleep duration and falls: a systematic review and meta-analysis of observational studies. $J$ Sleep Res 2017; 26(3):293-301.

29. Yoo IY. Recurrent falls among community-dwelling older Koreans: prevalence and multivariate risk factors. J Gerontol Nurs 2011; 37(9):28-40.

30. National Institute on Alcohol Abuse and Alcoholism (NIAAA). NIAAA releases physicians' guide [Internet]. Washington: NIAAA; 1995. Disponível em: https:// www.niaaa.nih.gov/news-events/news-releases/niaaa -releases-physicians-guide

31. World Health Organization (WHO). Recommended population levels of physical activity for health in Global Recommendations on Physical Activity for Health [Internet]. Genebra: WHO; 2010 [acessado 2018 Maio 1]. Disponível em: http://apps.who.int/iris/bitstream/ handle/10665/44399/9789241599979_eng.pdf;jsessionid=3CF6B32C2EF6901F0A3A0B1C638DE915? sequence $=1$ 
32. Lipschitz DA. Screening for nutritional status in the elderly. Prim Care 1994; 21(1):55-67.

33. Finch CF, Stephan K, Shee AW, Hill K, Haines TP, Clemson L, Day L. Identifying clusters of falls-related hospital admissions to inform population targests for prioritising falls prevention programmes. Inj Prev 2015; 21(4):254-259.

34. Perracini MR, Ramos LR. Fatores associados a quedas em uma coorte de idosos residentes na comunidade. Rev Saúde Pública 2002; 36(6):709-716.

35. Pinheiro MM, Ciconelli RM, Martini LA, Ferraz MB. Risk factors for recurrent falls among Brazilian women and men: the Brazilian Osteoporosis Study (BRAZOS). Cad Saúde Pública 2010; 26(1):89-96.

36. Gotzmeister D, Zecevic AA, Klinger L, Salmoni A. People are getting lost a little bit: systemic factors that contribute to falls in Community-dwelling octogenarians. Can J Aging 2015; 34(3):397-410.

37. Peel NM, McClure RJ, Hendrikz JK. Psychossocial factors associated with fall-related hip fractures. Age Ageing 2007; 36:145-151.

38. Hokby A, Reimers A, Laflamme L. Hip fractures among older people: do marital status and type of residence matter? Public Health 2003; 117:196-201.

39. Çakar E, Durmus O, Dinçer U, Kiralp MZ, Cerit-Soydan $F$. The effect of marital status on health quality and fall risk of elderly people. Turkish $J$ Geriatrics 2011; 14(4):331-336.

40. August KJ, Sorkin DH. Marital status and gender differences in managing a chronic illness: the function of health-related social control. Soc Sci Med 2010; 71(10):1831-1838.

41. Harmsen AMK, Egea-Gámez RM, Garssen FP, Dulken EV, Toor A, Bloemers FW. Fall-related injuries in Amsterdam: frail older at risk. J Women Aging 2016; 28(6):489-497.

42. Milat AJ, Watson WL, Monger C, Barr M, Giffin M, Reid M. Prevalence, circumstances and consequences of falls among community-dwelling older people: results of the 2009 NSW Fall Prevention Baseline Survey. NSW Public Health Bulletion 2011; 22:43-48.

43. Tricco AC, Thomas SM, Veroniki AA, Hamid JS, Cogo E, Strifler L, Khan PA, Robson R, Sibley KM, MacDonald H, Riva JJ, Thavorn K, Wilson C, Holroyd-Leduc J, Kerr GD, Feldman F, Majumdar SR, Jaglal SB, Hui W, Straus SE. Comparisons of interventions for preventing falls in older adults: a systematic review and meta-analysis. JAMA 2017; 318(17):1687-1699.
44. Zaitune MPA, Barros MBA, César CLG, Carandina L, Galdbaum M, Alves MCGP. Fatores associados à prática de atividade física global e de lazer em idosos: Inquérito de Saúde no Estado de São Paulo (ISA-SP), Brasil. Cad Saúde Pública 2010; 26(8):1606-1618.

45. Perracini MR, Teixeira LF, Ramos JLA, Pires RS, Najas MS. Fall-related factors among less and more active older outpatients. Braz J Phys Ther 2012; 16(2):166172.

46. Coutinho ESF, Silva SD. Uso de medicamentos como fator de risco para fratura grave decorrente de queda em idosos. Cad Saúde Pública 2002; 18(5):1359-1366.

47. Rodrigues MAP, Facchini LA, Piccini RX, Tomasi E, Thumé E, Silveira DS, Siqueira FV, Paniz VMV. Uso de serviços básicos de saúde por idosos portadores de condições crônicas. Rev Saúde Pública 2009; 43(4):604-612.

48. Liu HH, Eaves J, Wang W, Womack J, Bullock P. Assessment of canes used by older adults in senior living communities. Arch Gerontol Geriatr 2011; 52(3):299303.

49. Stevens JA, Thomas K, Teh L, Greenspan AI. Unintentional fall injuries associated with walkers and canes in older adults treated in U.S. emergency departments. J Am Geriatr Soc 2009; 57(8):1464-1469.

Artigo apresentado em 10/08/2018

Aprovado em 05/03/2019

Versão final apresentada em 07/03/2019

Editores chefes: Romeu Gomes, Antônio Augusto Moura da Silva 